\title{
Universiteit
}

Leiden

The Netherlands

\section{Towards a complete theory of high T-c}

Zaanen, J.; Chakravarty, S.; Senthil, T.; Anderson, P.; Lee, P.; Schmalian, J.; ... ; Rice, M.

\section{Citation}

Zaanen, J., Chakravarty, S., Senthil, T., Anderson, P., Lee, P., Schmalian, J., ... Rice, M. (2006). Towards a complete theory of high T-c. Nature Physics, 2(3), 138-143. Retrieved from https://hdl.handle.net/1887/5125

Version: $\quad$ Not Applicable (or Unknown)

License:

Downloaded from: https://hdl.handle.net/1887/5125

Note: To cite this publication please use the final published version (if applicable). 


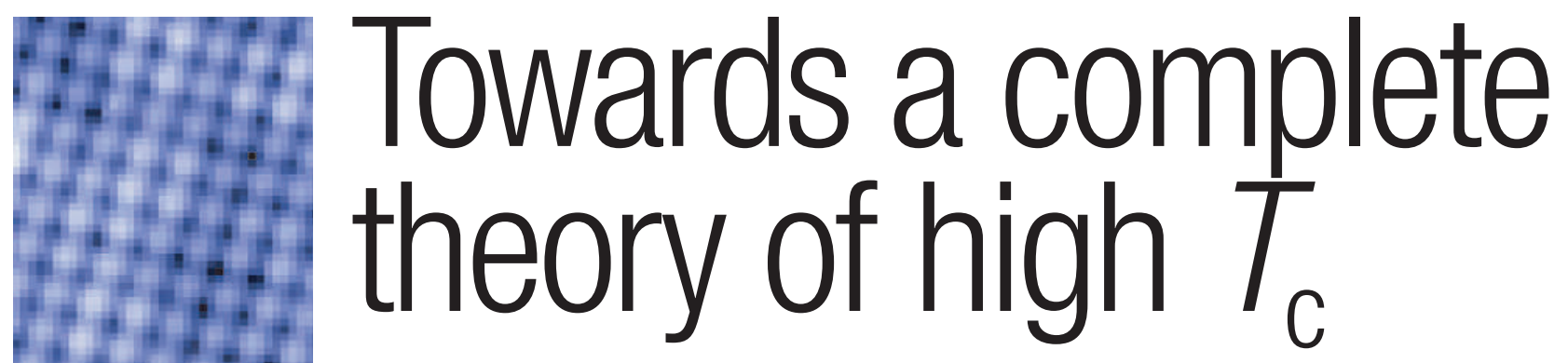

Given the successes of the microscopic theory of conventional superconductors, it seems natural to expect a similar all-encompassing theory for high-temperature superconductivity. But is it the best approach? Where are we heading?

s high-temperature superconductivity (high$T_{c}$ ) on the list of the most profound physics problems, or is it a collective illusion rooted in sociological developments in the late 1980s? It is not an illusion - I perceive high- $T_{\mathrm{c}}$ on a par with dark energy, extra dimensions and the secret of life.

High- $T_{c}$ is a graveyard of theories. However, this has had the beneficial effect of causing a state of mind not dissimilar from the goal of Zen Buddhism: think nothingness, in order to reach enlightenment. Rid the mind from textbook wisdoms, such as the conventional theory of Bardeen, Cooper and Schrieffer (BCS), the Fermi gas and even resonating valence bonds (RVBs), and the essence of high- $T_{\mathrm{c}}$ comes into view. The metallic state at optimal doping embodies the enlightenment. Rather than being complicated, this 'bad' metal shows a sacred simplicity symbolized, for example, by its linear resistivity as a function of temperature, up to the melting point of the crystal. This reveals principle at work, of a quality of Einstein's principle of equivalence.

I am convinced that this principle cannot be found by merely thinking hard — the reason I stopped designing theories. It requires experiments to give away the clue, and there are reasons to be optimistic. A group of experimentalists are providing further evidence that phonons play an important, albeit quite unusual, microscopic role. Even if this does not lead to the magic bullet, it has at least the effect of discrediting the influential religious dogma that the electronic toy models invented by Anderson and Hubbard will solve all problems.

The experimental characterization of the ordering phenomena competing with the superconductivity at low doping is rapidly improving. A growing body of evidence points at stripe order, though I see stripes as more of a convenience than a cause: the mysterious stuff responsible for the superconductivity and the bad metal comes to a standstill in stripy patterns, enabling experimentalists to have a closer look. The case currently emerging from neutron scattering and scanning tunnelling microscopy (STM) is in this regard highly promising.

There is one candidate for the Great Principle: quantum criticality, which has unlimited resources to make things simple. Unfortunately, the empirical evidence on its behalf is thin at best. I propose two demanding experiments: high-precision measurements of the chemical potential as a function of doping and temperature to catch the elusive quantum criticality, and a high-resolution measurement at small but finite momenta of the electron energy-loss function to look for the dual shear Higgs boson that is the unique signature of strong stripe correlations in the superconductor.

JAN ZAANEN

\footnotetext{
$\mathbf{P}$
}

hysicists are reductionists at heart. Otherwise, our science would be metaphysics. This is not an indictment on emergence, but a perspective that dates back to Newton. Before the BCS theory of conventional superconductivity, would you have thought that a microscopic mechanism of superconductivity existed? Being hardly in existence then, I cannot tell for sure. Yet, the history of science indicates that perhaps there was a wide sense of despair. Do I believe that there is a microscopic theory of high-temperature superconductuctivity? Undoubtedly, yes. Do I believe there is an ultimate microscopic theory, or a final theory of superconductivity? Undoubtedly, no. This is amply clear from the success of the BCS mechanism of conventional superconductors, and, at the same time, from its dramatic failure for high-temperature superconductors.

In certain circles it is fashionable to ignore that the transition temperature itself is the driving force of this field, and to emphasize instead the so-called universal properties of matter. Yet, the dream of room-temperature superconductivity is the secret motivating factor. Think back to the mechanism of 
magnetism. As I mentioned elsewhere (Science 266, 386-387; 1994), "although magnets with extremely high magnetic transition temperatures, such as iron, were known to Phoenician sailors, or even earlier, the understanding of the mechanism is a 20th-century post-quantum-mechanical achievement." Maybe a similar major revolution will be necessary.

Where should we place our emphasis? I believe that the key is the multilayer copper oxide materials for which transition temperatures within a homologous series rise and fall like a dome with the number of copper oxide layers. The rise can be as large as $45 \mathrm{~K}$ from a single-layer compound to a three-layer compound. Considering that systematic, sustained efforts never led to transition temperatures higher than $23.2 \mathrm{~K}\left(\mathrm{Nb}_{3} \mathrm{Ge}\right)$ in conventional superconductors, this is truly sobering. Why should such a thing ever happen?

As we muse about the secret of superconductivity, we shall continue to revel in the discovery of novel competing orders, and wonder if there is truly an allelectronic mechanism of superconductivity.

SUDIP CHAKRAVARTY

$t$ is useful to divide the issues in the field

based on the phase diagram. There is the superconductivity itself and the parent Mott insulator, but more mysterious perhaps are the pseudogap state and the strange optimally doped metal. The challenge is to find a theory that is rooted in the microscopics of the copper oxides, which is able to account for robust features of the physics that are common to all of them. I believe such a theory will eventually be found.

Impressive progress has been made over the years. The importance of the proximity to

\section{THE SUPERCONDUCTIVITY ITSELF SEEMS AMONG THE LEAST MYSTERIOUS OF THE VARIOUS PHENOMENA}

a Mott insulator for both the pseudogap and the superconductivity has become quite well established. Remarkably the superconductivity itself seems among the least mysterious of the various phenomena in the copper oxides. There are a number of theoretical demonstrations, at various levels of rigour, of the possibility of superconductivity in doped Mott insulators.

At a qualitative level, the origin of the normalstate pseudogap also seems reasonably clear - as a spin gap formed by singlet formation between electrons that are localized long enough for the magnetic superexchange to do its job. However, a detailed description of the pseudogap region is still out of reach. But there are plenty of reasons to hope that it may be just around the corner.

Most mysterious is the strange optimally doped metal. There simply do not seem to be (m)any good ideas on how to think about it. Yet it is empirically characterized by simply stated laws.
Perhaps some conceptual advance is needed to get a theoretical description of it.

As to where we are heading, I think there is plenty of reason to be optimistic. I imagine that the physics of the pseudogap and the superconductivity will be further clarified enormously in the next few years. Hopefully this will set the stage for serious theories to emerge on the optimally doped metal. Perhaps we will then also understand somewhat similar 'strange metal' physics in other systems, such as the heavy fermions near critical points.

An important spin-off from work on the high$T_{\mathrm{c}}$ theory has been the emergence and clarification of a number of new theoretical ideas, some of which have become entirely separate research areas. Some of these could possibly greatly impact the study of correlated materials vastly different from the copper oxides.

\section{T. SENTHIL}

t has been my (published) opinion for years that the cause of high-temperature superconductivity is no mystery. We now have a workable theory - not just for calculating the broad outlines (the transition temperature $T_{\mathcal{c}}$, energy-gap shape, effect of doping, pseudogap temperature) but details of the anomalous phenomenology. A crude version of this theory was published in 1988 by Zhang and co-authors (Supercond. Sci. Technol. 1, 36-38; 1988), based partly on my earlier ideas, and in a similar paper, Kotliar and Liu came to the same conclusions independently (Phys. Rev. B 38, 5142-5145; 1988). But the successes weren't then recognized because experiments were too primitive. The theory was revived with modern precision five years ago by Sorella and by Randeria and co-workers, and recently I have added some details, enabling calculations of such anomalies as asymmetric tunnelling spectra and their doping dependence, the 'Cheshire cat' disappearance of the Fermi surface and the strange pseudogap phenomena. It also answers such deeper questions as 'why these materials?' and 'why is the gap real?'.

The theory is an RVB theory in the sense that it is based on the Gutzwiller projection of a conventional (BCS) singlet-pair wavefunction, but uses the $d$-wave version of Kotliar, for example. Otherwise it is mostly simple mean-field BCS. The essential physics is the doped Mott insulator obeying the $t-J$ hamiltonian, in which the hopping matrix elements of $t$ to states having high energy $U$ are removed in favour of an antiferromagnetic exchange interaction $J$. My new gimmick is a fugacity parameter, which allows pairs to vary their relative holon-pair to spinonpair character with doping, momentum around the Fermi surface, or position. There are still interesting questions to be answered, but in my opinion this brings us to the BCS stage of understanding.

The role of experiment cannot be overstated. Most recent and useful has been the flowering of STM under Davis and co-workers, but equally fundamental have been the precision measurements by Ong and his students of bizarre transport and equilibrium phenomena, with the help of many

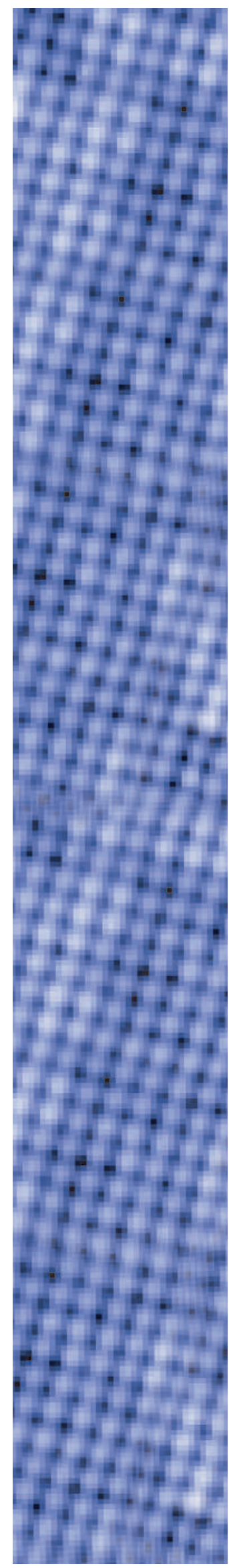




\section{FE THE BRINGS US TO THE BCS STAGE OF UNDERSTANDING}

crystal-growers - it is unfair but probably correct to single out Liang, Bonn and Hardy at the University of British Columbia. Developments in precision angleresolved photoemission spectroscopy are also worth mentioning, with the Stanford and Argonne groups leading. Infrared spectroscopy is important also. What I would ask for is more of the same - if high$T_{\mathrm{c}}$ does nothing else, the experimental cornucopia it has fostered will have been worth it!

\section{PHILIP ANDERSON}

l: took ten years of intense experimental effort to establish that high- $T_{\mathrm{c}}$ is a genuinely new phenomenon. I have in mind the phenomenology of the underdoped pseudogap state, with a myriad of new phenomena unlike anything we have seen before. By now I think there is broad agreement that 'doping into a Mott insulator' is the correct and requisite microscopic starting point. In a recent review with Naoto Nagaosa and Xiao-Gang Wen (Rev. Mod. Phys. 78, 79-86; 2006), we made two key points: (1) Anderson's RVB theory contains the central idea that naturally explains the new physics - that is, the tendency of quantum spin- $1 / 2$ to form singlets, and that doping of such singlets lead to superconductivity. (2) The mathematical expression of these ideas is the gauge theory and all its emergent properties.

What about the next decade? As with any genuinely new idea, it will take time to convince the sceptics, who rightfully demand to know: Where is the gauge field? Where is the spinon? It is indeed the job of the advocates to answer such questions. A recent proposal by Senthil and me on an experimental setup to generate and detect gauge flux is a step in that direction. At the same time, we will see further development of the notion of emergence of gauge fields and fractionalized particles as lowenergy phenomena in systems that did not contain them in the starting model. The past five years have seen great progress in this direction. Thanks to the work of Kitaev, Wen, Senthil and others, we now know of several models that realize such emergence. We will need to create more realistic models and hopefully real experimental systems, so that the idea of an emergent gauge field enters the vocabulary of condensed-matter physics, just as the emergence of a Goldstone boson entered the vocabulary of critical phenomena several generations earlier.

Finally, it will help if we can demonstrate superconductivity in a purely repulsive Hubbard model. This will mostly likely be achieved by numerical methods, but I believe it will be done.

PATRICK LEE

$T$ he search for the theory of high- $T_{c}$ superconductivity is wide open. Still, there is strong evidence that the pairing mechanism is predominantly electronic, that some kind of quantum spin dynamics plays a key role, and that the physics of the Mott insulator of the parent compounds remains important even as the material becomes superconducting.

The biggest mystery is the linear resistivity at optimal doping. Although there are numerous competing theories for pseudogap behaviour and $d$-wave pairing, it is extremely hard to formulate a single sound microscopic theory of the transport properties. Maybe another puzzle of fundamental importance is the dependence of $T_{\mathrm{c}}$ on the number of layers.

To develop the theory of copper-oxide superconductors one could take two extreme positions: (1) the numerous unconventional properties are due to a fortuitous coincidence of several unrelated effects (for example, there is one theory for the transport properties that is consistent with but not fundamentally connected to the explanation of the mechanism), or (2) there is a natural explanation for all the puzzles in a new theory for correlated quantum matter.

Although both possibilities in principle lead to further optimization of the materials, and maybe even a room-temperature superconductor, the systematic

\section{THE SEARCH FOR THE THEORY OF HIGH- $T_{C}$ SUPERCONDUCTIVITY IS WIDE OPEN.}

phenomenology of several materials strongly supports the second view. This is the most important reason to continue working on this problem!

Which topics should be high on our priority list for future research? We need to better understand what a Mott insulator is, and to learn from other systems (f-electron superconductors, organic superconductors). We should also develop better local probes, such as double-tip STM to study electron interference, and combinations of nuclear magnetic resonance and STM.

\section{JÖRG SCHMALIAN}

S ince initial studies of the copper-oxide superconductors, it has been well-recognized that the superconductivity occurs in doped Mott insulators near the Mott transition. However, the criticality of the Mott transition was not clearly identified until recently, so the crucial role of the Mott criticality for the mechanism of superconductivity has been under-appreciated. In fact, the Mott transition is driven by the order parameter of electron density (charge degrees of freedom) accompanied by critical density fluctuations at small wavenumbers, and has nothing to do with the magnetic degrees of freedom by themselves.

Although magnetic fluctuations have been studied extensively as possible pairing glue, simply owing to their relatively easy accessibility by theoretical tools - in the range from weak coupling 
to strong correlation regimes described by the so-called $t-J$ model - such fluctuations are just one of many consequences of competing orders revealed in various transition metal oxides in the proximity of a Mott insulator. Strong correlations and enhanced magnetic fluctuations are commonly observed in this family of materials, but layered copper oxides are unique in showing such high transition temperatures. No theory has been able to explain why most of the doped or metallized antiferromagnetic insulators such as $\mathrm{LaTiO}_{3}, \mathrm{~V}_{2} \mathrm{O}_{3}$, $\mathrm{NiS}_{2}$ and $\mathrm{Sr}_{2} \mathrm{VO}_{4}$ do not show superconductivity even at very low temperatures. Neither the relatively large antiferromagnetic superexchange interaction nor strong two-dimensionality has been able to explain such a dramatic difference convincingly.

An ultimate microscopic theory of the high- $T_{c}$ superconductors will emerge after clarifying how the Mott transition and its proximity, namely the mother transition of many other competing orders, is so special in these materials. The difficulty over these past twenty years partly comes from the lack of good experimental probes for dynamical and short-range density (charge) correlations - although some signatures of static density fluctuations have been observed in STM, for instance. In contrast to magnetic correlations studied by neutron scattering and NMR, probes for wavenumber- and frequencydependent charge correlations, such as electron energy-loss spectroscopy or X-ray spectroscopy, are still too poor to reveal the strong but diffusive charge fluctuations expected in the Mott proximity - its dielectric fluctuation energy should extend to $1 \mathrm{eV}$. A breakthrough of the resolution in such experimental probes will solve the long-standing puzzle.

MASATOSHI IMADA

T he mechanism of high- $T_{\mathrm{c}}$ superconductivity is purely electronic - superconductivity without phonons - arising from a magnetic interaction between quasiparticles reflecting their close approach to antiferromagnetism. The same mechanism is at work in the organic and heavy-electron superconductors, with the possible exception of the second superconducting transition in $\mathrm{CeCu}_{2}\left(\mathrm{Si}_{1-x} \mathrm{Ge}_{x}\right)_{2}$ under pressure.

Direct experimental confirmation of the intimate relationship between the build-up of antiferromagnetic correlations and the superconducting transition has come from a recent analysis by Nick Curro of experiments on the spin-lattice relaxation rate $1 / T_{1}\left(T_{1} T\right.$ provides a direct measure of the build-up of antiferromagnetic correlations) in three materials with very different superconducting transition temperatures: optimally doped $\mathrm{YBa}_{2} \mathrm{Cu}_{3} \mathrm{O}_{7-\delta}\left(T_{c}\right.$ $\sim 90 \mathrm{~K}), \mathrm{CeCoIn}_{5}\left(T_{\mathrm{c}} \sim 2 \mathrm{~K}\right)$ and $\mathrm{PuCoGa}_{5}\left(T_{\mathrm{c}} \sim 18\right.$ $\mathrm{K})$. Curro and co-authors find that $T_{1} T$ scales with the superconducting transition temperature (Mater. Res. Soc. Bull. 30, 442-446; 2005).

For the underdoped materials exhibiting pseudogap behaviour, the theory is some years away. For these materials, beginning with the pioneering work of David Johnston, experiments on their magnetic properties have shown that there are two distinct components present - a 'spin liquid' component and a Fermi liquid component. What we do not currently know is the nature of the 'normal' state out of which superconductivity arises in these underdoped materials. Is it spatially homogeneous, in which case the two components refer to different parts of the Fermi surface, or is it, at least on dynamic timescales, intrinsically inhomogeneous?

Further experimental probes need to characterize completely the low-frequency 'protected' behaviour in both the normal and superconducting states of an overdoped superconductor (a transition temperature of 70 $\mathrm{K}$ or higher). This material would be the 'poster child' for materials in which pseudogap behaviour (which normally appears below a temperature $T^{\star}$ ) does not raise its ugly head. Then the same approach should be applied to a number of the underdoped superconductors.

The study of the remarkably rich and unexpected behaviour of the copper oxides will continue to yield surprises. I think we are close to a theoretical consensus on the magnetic origin of both pseudogap and superconducting behaviour, and that the presence of a spin-liquid with a pseudogap is not good for superconductivity. It is a foe not a friend. There may continue to be a community reluctance to admit, in public at least, that for overdoped materials we are close to having a consistent microscopic theory, for fear, perhaps, of thereby losing some funding.

There will continue to be little theoretical consensus on the nature of the spin-liquid at temperatures below $\sim T^{\star} / 3$, where it seems to become gapped. Does it become a new state of matter, with a well-defined order parameter? If so, which of the many candidates for this state is the right one? Here is where so much of the present and likely future action is and will be found.

DAVID PINES

$T^{t}$ he problem of high- $T_{\mathrm{c}}$ superconductivity is in fact a set of problems related to the unusual phases, phase transitions and crossovers in a quasi-two-dimensional doped Mott insulator. These materials challenge three basic paradigms of 20th-century solid-state physics: band theory, Landau's Fermi liquid theory and the BCS theory of superconductivity. This, together with the absence of small parameters, has made progress on high- $T_{c}$ theory difficult.

\section{THE PROBLEM OF HIGH- $T_{\mathrm{C}}$ SUPERCONDUCTIVITY IS IN FACT A SET OF PROBLEMS}

The present status of high- $T_{c}$ theory can be summed up in one sentence: the superconducting state is now understood in detail, but the abnormal 'normal' states - the pseudogap and especially the strange metal - are much less understood.

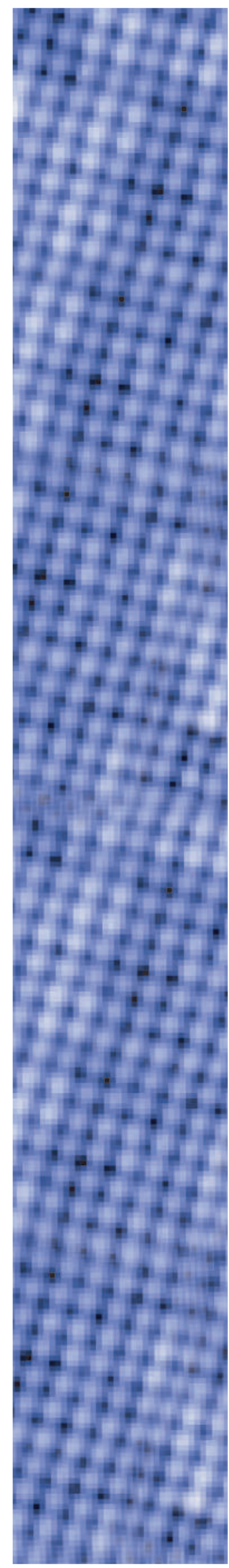




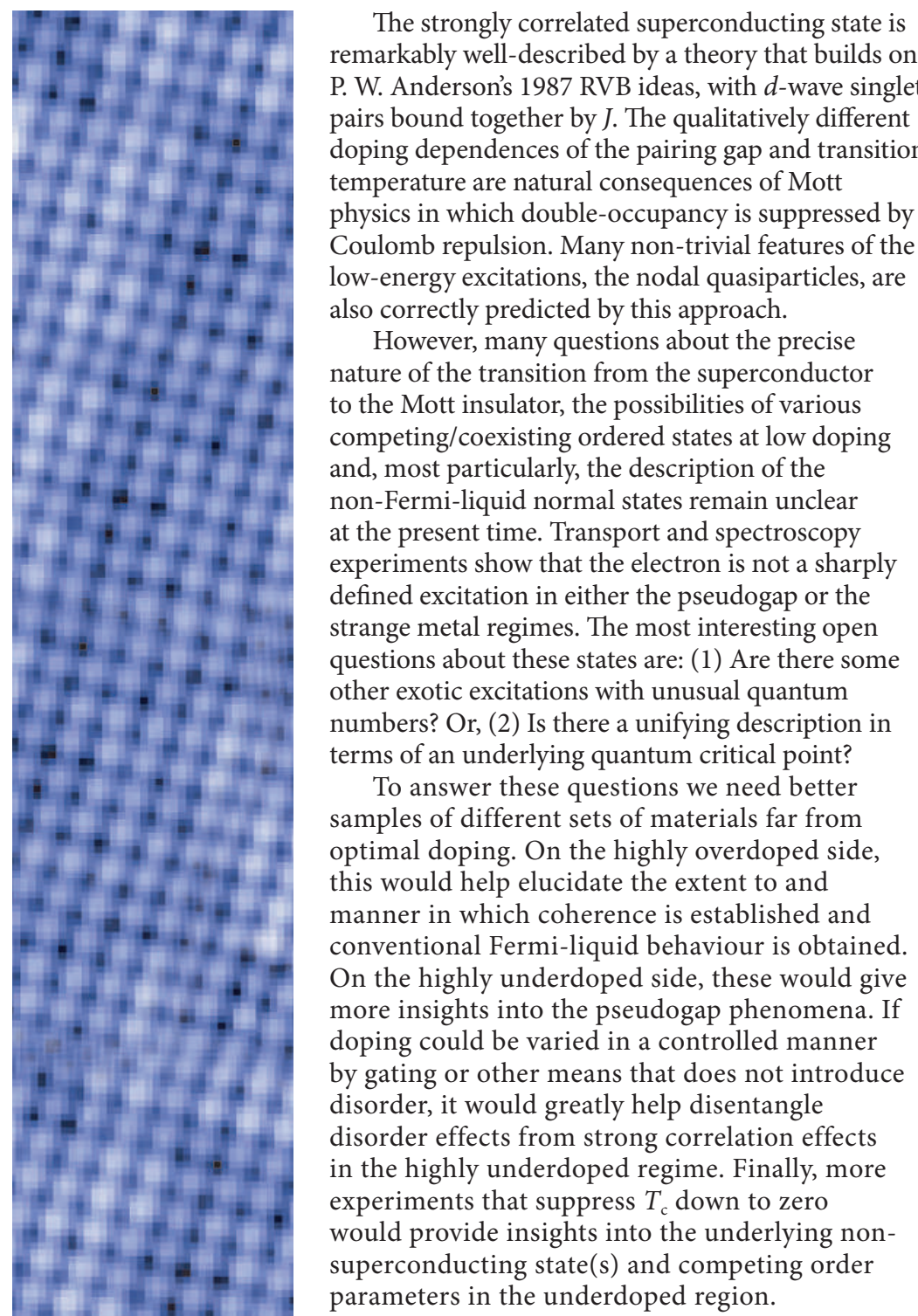

MOHIT RANDERIA

A recent tour de force experiment (B. Fauqué et al. Preprint at <http://arxiv.org/abs/ cond-mat/0509210>; 2005) verifies the crucial prediction of a microscopic theory of high- $T_{c}$ phenomena. With the chemistry of the copper oxides in mind, I predicted that the pseudogap region in the phase diagram of the copper oxides violates time-reversal symmetry through spontaneous current-loops in two-dimensional $\mathrm{O}-\mathrm{Cu}-\mathrm{O}$ plaquettes. Furthermore, the scaleinvariant fluctuations about the quantum critical point (QCP) where the pseudogap ends provide the glue for pairing, as well as lead to the anomalous normal state properties. The QCP and the fluctuations were anticipated by the marginal Fermi-liquid phenomenology, which has been tested extensively.

The lynch-pin of the theory is the broken symmetry of the pseudogap phase. This has now been directly determined through polarized neutron diffraction. In four different underdoped
$\mathrm{YBa}_{2} \mathrm{Cu}_{3} \mathrm{O}_{6+\delta}$ samples, Fauqué et al. found magnetic scattering at the Bragg peaks, which is consistent only with the predicted symmetry, and with the interpretation (M. E. Simon and C. M. Varma. Phys. Rev. Lett. 89, 247003; 2002) of the observation (A. Kaminski et al. Nature 416, $616-613$; 2002) of dichroism in angle-resolved photoemission in underdoped $\mathrm{Bi}_{2} \mathrm{Sr}_{2} \mathrm{CaCu}_{2} \mathrm{O}_{8+\delta}$. In both experiments the new features disappear above the pseudogap temperature.

These experiments and the theory answer the two major questions posed by the high- $T_{\mathrm{c}}$ superconductors: What is the correct model hamiltonian that gives the universal features of the observed phase diagram? And what is the nature of the fluctuations that are the source of marginal Fermi-liquid and the glue for superconductivity? Current loops within a unit-cell require at least three dynamical degrees of freedom as well as $\mathrm{Cu}-$ $\mathrm{O}$ interactions in the proposed hamiltonian. The experiments confirm that time-reversal invariance is broken in the pseudogap phase ending at a QCP. Consistent with the general theory of QCPs, the fluctuations are then the fluctuations of time that condense to form the circulating currents in the pseudogap phase.

Twenty years after the discovery and after the most intense activity on any scientific problem in history, these and other excellent experiments may have identified the correct theory of the high- $T_{\mathrm{c}}$ phenomena.

CHANDRA VARMA

W hy is $T_{c}$ so high and how can we make it higher? That is probably the ultimate question in the context of high$T_{\mathrm{c}}$ superconductivity. Answering it requires a thorough understanding of the pairing mechanism. Interestingly, the superconducting state - at least at low temperatures - seems

\section{THE DIFFICULTY IN THE FIELD DOES NOT LIE IN FINDING ANSWERS BUT IN FORMULATING SMOKING- GUN QUESTIONS}

conventional in that most of its properties seem to be well-described by $d$-wave BCS theory. Even the pairing mechanism is one of the less-controversial aspects: spin fluctuations are very likely to play a key role, although phonons may also participate.

The difficulties lie with the normal state, featuring phenomena like the apparent linearin-temperature resistivity at optimal doping, multiple pseudogap scales in the underdoped regime, competing phenomena associated with translational symmetry breaking, such as stripes or checkerboard states, and the thermal transport properties in the vicinity of the transition to the Mott insulator, to name a few. Although various explanations for these observations 
have been proposed, a consistent picture has not yet emerged.

But do we need to understand these phenomena to understand high- $T_{\mathrm{c}}$ superconductivity? Are stripes important or just a secondary issue unrelated (and detrimental) to the pairing? Are the strong inhomogeneities seen by STM a key property or an unimportant surface effect? Is it mandatory to understand the pseudogap in order to design materials with higher $T_{c}$ ?

The answer may be yes or no depending on whether the normal or the superconducting phase is viewed as the basis for the theoretical attack. What level of understanding is needed to solve the high- $T_{\mathrm{c}}$ problem? Remarkably, the difficulty in the field does not lie in finding answers but in formulating smoking-gun questions - both to experiment and theory.
Progress will only be made by discerning primary from secondary effects.

\section{MATTHIAS VOJTA}

W hen it comes to 'the theory' and the RVB model, I'm a believer. But though RVB can explain a lot (see the recent topical review by $\mathrm{P}$. W. Anderson et al. J. Phys. Condens. Matter 16, R755-R769; 2004) there is much to be done to develop a full theory that describes the anomalous 'pseudogap' normal phase at underdoping and eventually the crossover to the standard Landau Fermi-liquid behaviour at overdoping. Experiments on clean well-ordered underdoped cuprates will be a key to progress, and these are appearing at last, thanks to the Ortho-II $\mathrm{YBa}_{2} \mathrm{Cu}_{3} \mathrm{O}_{6.5}$ samples grown by the University of British Columbia group. So I am optimistic!

MAURICE RICE

\section{The contributors}

Jan Zaanen is at the Instituut-Lorentz, Universiteit Leiden, 2300 RA Leiden, The Netherlands. e-mail: jan@lorentz.leidenuniv.nl

Sudip Chakravarty is in the Department of Physics and Astronomy, University of California, Los Angeles, California 90095-1547, USA.

e-mail: sudip@physics.ucla.edu

T. Senthil is in the Department of Physics, Indian Institute of Science, Bangalore 560 012, India. e-mail: senthil@physics.iisc.ernet.in

Philip Anderson is in the Department of Physics, Princeton University, Princeton, New Jersey 08544, USA.e-mail: pwa@pupgg.princeton.edu

Patrick Lee is in the Department of Physics, Massachussetts Institute of Technology, Cambridge, Massachussetts 02139-4307, USA.

e-mail: palee@mit.edu

Jörg Schmalian is in the Department of Physics and Astronomy and Ames Laboratory, Iowa State University, Ames, Iowa 50011, USA. e-mail: schmalian@ameslab.gov

Masatoshi Imada is at the Institute for Solid State Physics, University of Tokyo, Kashiwa, Chiba 2778581, Japan.e-mail: imada@issp.u-tokyo.ac.jp
David Pines in the Department of Physics,

University of Illinois at Urbana-Champaign, Urbana, Illinois 61801-3080, USA.

e-mail david.pines@comcast.net

Mohit Randeria is in the Department of Physics, Ohio State University, Columbus, Ohio 43210, USA. e-mail: randeria@mps.ohio-state.edu

Chandra Varma is in the Department of Physics, University of California, Riverside, California 92521, USA.e-mail: chandra.varma@ucr.edu

Matthias Vojta is at the Institut für Theorie der Kondensierten Materie, Universität Karlsruhe, 76128 Karlsuhe, Germany.

e-mail: vojta@tkm.physik.uni-karlsruhe.de

Maurice Rice is at the Institut für Theoretische Physik, Eidgenössische Technische Hochschule Zürich, CH-8093 Zürich, Switzerland. e-mail: rice@itp.phys.ethz.ch

Image: Atomically resolved scanning tunnelling microscope image of the surface of $\mathrm{Ca}_{2-}$ ${ }_{x} \mathrm{Na}_{x} \mathrm{CuO}_{2} \mathrm{Cl}_{2}$, showing a clear 'checkerboard' pattern. The spatial correlations indicate the presence of underlying order in the pseudogap state. Reprinted with permission from T. Hanaguri et al. Nature 430, 1001-1005 (2004).

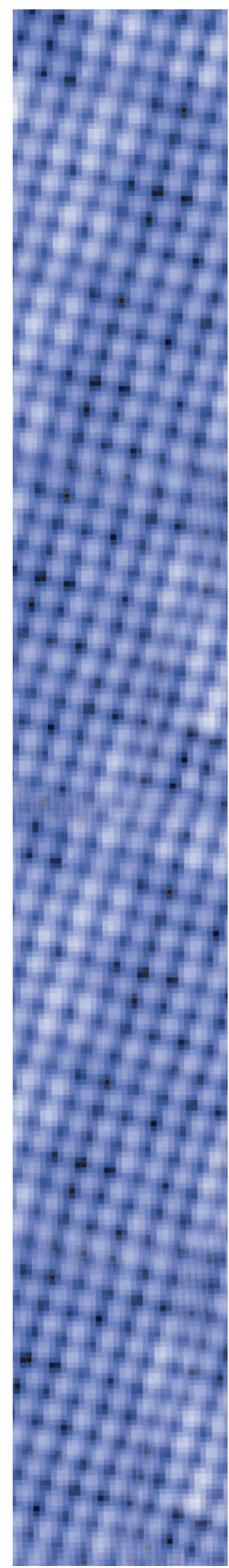

